\title{
Effect of Changes in Food Consistency on NADH-Ubiquinone Oxidoreductase Activity and Levels of mRNA for ND1, 51kDa, 75kDa and Myosin Heavy Chain Isoforms in Two Different Portions of Rat Masseter Muscle
}

\author{
By \\ Yoshiaki IDE and Iwao SATO \\ Department of Anatomy, School of Life Dentistry at Tokyo, the Nippon Dental University, 1-9-20 Fujimi, \\ Chiyoda-Ku, Tokyo 102-8159, Japan
}

- Received for Publication, February 14, $2006-$

\begin{abstract}
Key Words: NADH-ubiquinone oxidoreductase activity ND1, 51kDa, 75kDa, Myosin heavy chain isoform, Masseter muscle

Summary: We investigated the effect of a change in food consistency on properties of the masseter muscle in 3-week-old rats fed a soft diet for 9 weeks (Group S) and fed a soft diet for 5 weeks followed by a hard diet for 4 weeks (Group S-H). The NADH-O ${ }_{2}$ oxidoreductase activity, levels of mRNAs transcribed from genes encoding NADH-ubiquinone oxidoreductase (Complex I: ND1, 51kDa, and 75kDa) and myosin heavy chain (MyHC) isoforms and the phenotype of the muscle fibers were measured in the superficial and deep portions of the muscle. In the period from 8 weeks to 12 weeks of age, $\mathrm{NADH}-\mathrm{O}_{2}$ oxidoreductase enzyme activity in both the superficial and deep portions of the muscle showed similar patterns in Group S and Group S-H. In contrast, the ND1, 51kDa and 75kDa mRNA levels in the superficial and deep portions of the masseter muscle in the Group S-H were higher than those of Group $S$ in the 12-week-old rats, except for the 51kDa mRNA in the superficial portion of the masseter muscle. MyHC-IIa and MyHC-IId/x mRNA levels in the superficial portion of the masseter muscle were higher in the Group S-H than in the Group S. These observations suggest that short-term feeding stress such as the transition from a soft diet to a hard diet causes changes in oxidative metabolism, in mRNA levels for the Complex I components ND1 and 75kDa, and the mRNA levels for the $\mathrm{MyHC}$ isoforms IIa and IId/x in the superficial portion of rat masseter muscle, but no changes in the composition of muscle fiber types.
\end{abstract}

The proportions of muscle fiber types and the types of myosin heavy chain (MyHC) isoforms in the masseter muscle and the metabolic properties of the muscle tissue have been shown to vary during postnatal life ${ }^{1-4)}$. The phenotype of the muscle fibers reflected the histochemical profile of various enzymes, such as cytochromes (b, c, c1, a and a3), F1-ATPase, succinate and NADH dehydrogenases on various depending on the diets and occlusal conditions $^{5-10)}$. The adaptive ranges of different muscle fibers are dependent on the bite-raising conditions of the masseter muscle ${ }^{11)}$. Histochemical staining for NADH dehydrogenase activity allows muscle fibers to be classified into 3 types (Type I, strong reaction; Type IIA, intermediate reaction; Type IIB, weak reaction) and these types are re- lated to the localization volume of mitochondria in the muscle fiber. Complexes I, II, III and IV are respiratory chain enzymes in the inner membrane of mitochondrial cristae. NADH-ubiquinone oxidoreductase (Complex I) transfers electrons from $\mathrm{NADH}$ to ubiquinone. Electrons from both succinate and NADH are sequentially transported via ubiquinone to several common enzymes, and finally $\mathrm{O}_{2}$ is reduced by cytochrome c oxidase. NADH- $\mathrm{O}_{2}$ oxidoreductase activity can be monitored with an oxygen electrode, which allows the monitoring of net electron transfer from $\mathrm{NADH}$ to $\mathrm{O}_{2}{ }^{12)}$. Overall electron transport activity is closely related to the physiological function of the energy-supplying system of mitochondria. NADH dehydrogeanase is encoded by both the nuclear and the mitochondrial

Correspondence to: Iwao Sato, Department of Anatomy, School of Life Dentistry at Tokyo, the Nippon Dental University, 1-9-20 Fujimi, Chiyoda-ku, Tokyo 102-8159, Japan. E-mail: iwaoa1@tokyo.ndu.ac.jp 
genomes. During postnatal development of the rat masseter muscle, expression of ND1 (mitochondrial genome) mRNA and $51 \mathrm{kDa}$ (nuclear genome) mRNA differs depending on feeding behavior from suckling to mastication ${ }^{13)}$. Both NADH dehydrogeanase enzyme activity and expression of ND1 mRNA and 51kDa mRNA are differed in rat tongue muscle ${ }^{14)}$. Different expressions of mRNA between nuclear and mitochondrial genomes reflect the profile of NADH dehydrogeanase enzyme activity in muscle fiber types of masseter muscle. The proportion of muscle fiber types (Type IIA and Type IIB) in the superficial portion of the rat masseter muscle changes under protrusion of mandible $^{7)}$. A smaller percentage of Type IIA fibers and a larger percentage of Type IIB fibers have been found in the anterior deep rat masseter muscle in a group fed a soft $\operatorname{diet}^{6)}$. The reduction of Type IIB fiber was found in profound of rat masseter muscle by occlusal contention and soft diet feeding ${ }^{9)}$. Mouse masseter muscle was composed of three layers (superficial, intermaediate and deep) defined by the tendons of origin or insertion, and there were differences in myosin heavy-chain isoforms in each layer ${ }^{15}$. The functional compartment in masseter muscle affect to the phenotype of the muscle fibers and enzyme activity under feeding mechanical stress. Therefore, changes in the histochemical profile of muscle fibers may reflect different requirements for energy metabolism resulting from changes in feeding behavior. However, the effects of feeding conditions in masseter muscle were obtained by occlusal conditions ${ }^{7,9,10,16,17)}$ and soft diet feeding ${ }^{6,18}$. We therefore investigated the functional compartment between the superficial and deep portions of the masseter muscle by using rats subjected to feeding conditions (soft diet to hard diet) as an experimental model. We measured changes in the morphological properties of the muscle fiber types and used a Clark-type electrode to measure dissolved oxygen as a means of estimating $\mathrm{NADH}-\mathrm{O}_{2}$ oxidoreductase activity. We also determined the transcription levels of various genes by RT-PCR analysis of expression of the mRNAs for Complex I components and $\mathrm{MyHC}$ isoforms.

\section{Materials and Methods}

\section{Animals and feeding conditions}

The male wistar rats were procured from the Nippon Medical Science Animal Resource Laboratory and bred at the Animal Testing Center of the Dept. of Life Dentistry, Nippon Dental University. Seventy-two rats were assigned to two groups (Group S and Group S-H). Group S rats were fed a

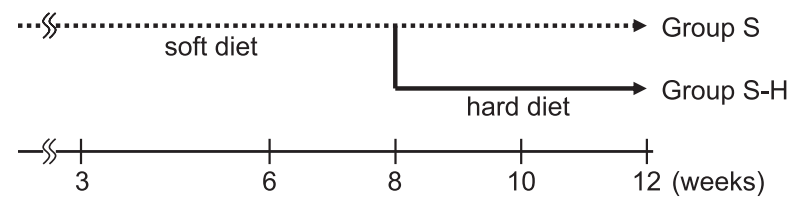

Fig. 1. Experimental design. The total duration of the experiment was 12 weeks. The rats in the Group S were fed a soft diet (3-12 weeks). The rats in the Group S-H were fed a soft diet (3-8 weeks) followed by a hard diet ( $8-12$ weeks).

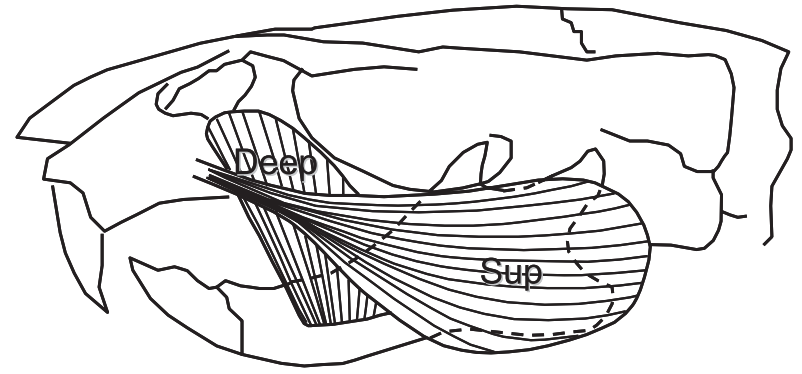

Fig. 2. Drawing of a lateral view of the left aspect of the rat masseter muscle. The muscle is composed of two layers (Sup, Superficial portion; Deep, Deep portion).

soft diet consisting of fine powder (MF powder, Oriental Yeast Inc., Tokyo, Japan) from 3 weeks to 12 weeks of age. Group S-H rats were fed a soft diet from 3 to 8 weeks of age and a hard diet consisting of solid diet pellet (MF, Oriental Yeast Inc., Tokyo, Japan) from 8 weeks to 12 weeks of age. They were bred under $24.1 \pm 1^{\circ} \mathrm{C}$ room temperature, $50 \pm 10 \%$ humidity, and 12 hours cycle of brightness. These animals were sacrificed by overadministration of pentobarbital followed by removal of the masseter muscle at 8,10 and 12 weeks of age (Fig. 1). Rat masseter muscle was composed of two layers (superficial and deep) defined by the tendon of origin or insertion (Fig. 2). Five specimens from the two groups at each stage (Fig. 1) were used for light microscopic study, assays and analysis of mRNA by RT-PCR.

\section{Light microscopic study}

Fresh samples of masseter muscle were prepared from four rats from each sample of the Group $\mathrm{S}$ $(\mathrm{n}=4$ at each age) and the Group S-H $(n=4$ at each age) (Fig. 1). The samples were immediately embedded in OCT compound (Tissue Tek, Sakura Finetechnical Co., Ltd., Tokyo, Japan) and frozen in liquid nitrogen. Frozen frontal sections (about $20 \mu \mathrm{m}$ thick) of the masseter muscle 
were cut with a cryostat. These sections were subsequently stained for NADH enzyme activity. They were examined under a light microscope (Vanox-S, Olympus Co., Tokyo, Japan) to classify muscle fibers into Type I (strong reaction), Type IIA (intermediate reaction) and Type IIB (weak reaction $)^{9,19)}$ and to measure their diameter. The diameter of the muscle fibers was identified by maximum of diameter of cross sectional muscle fiber in rat masseter muscle.

\section{Preparation of mitochondria}

Mitochondria were isolated by ultracentrifugation according to the method of Hogeboom $(1955)^{20)}$ with slight modifications. Immediately following the sacrifice of male Wistar rats at 8 , 10 and 12 weeks of age ( $\mathrm{n}=3$ at each age), $1 \mathrm{~g}$ of masseter muscle was minced in a buffer solution (Solution A, $10 \mathrm{ml}$ ) containing $0.25 \mathrm{M}$ sucrose, $0.1 \mathrm{mM}$ ethylenediamine tetra-acetic acid (EDTA), and $5 \mathrm{mM}$ Tris- $\mathrm{HCl}$ (pH 7.4) with a PotterElvehjen type homogenizer (Digital homogenizer, Iuchi, Osaka, Japan). After centrifuging the homogenate at $700 \times \mathrm{g}$ for $10 \mathrm{~min}$, the supernatant was centrifuged at $7,000 \times \mathrm{g}$ for $10 \mathrm{~min}$. The resulting sediment was washed once with Solution A and twice with $0.25 \mathrm{M}$ sucrose adjusted to $\mathrm{pH} 7.4$ with potassium hydroxide (Solution B). All procedures were performed at $0-4^{\circ} \mathrm{C}$.

\section{Enzyme assays}

Protein in rat masseter muscle mitochondria (3.0-10 $\mu \mathrm{g}$ of protein) was assayed by the Lowry method $^{21)}$ with bovine serum albumin as standard. $\mathrm{NADH}-\mathrm{O}_{2}$ oxidoreductase activity was measured polarographically with a Clark-type oxygen electrode (Yellow Spring Ltd. Ohio, USA). The oxidase activity assay with $50 \mathrm{mM}$ Tris- $\mathrm{HCl}(\mathrm{pH} 7.5)$ was performed at $25^{\circ} \mathrm{C}(258 \mathrm{nmole} / \mathrm{ml}$ dissolved oxygen) in a closed $2 \mathrm{ml}$ reaction chamber equipped with a magnetic stirrer and oxygen consumption was determined from the oxygen uptake curve on the chart recorder. Each reaction was initiated by the addition of NADH substrate $(500 \mu \mathrm{M})$.

\section{Isolation of total RNA}

Immediately following the sacrifice of male Wistar rats at 8,10 and 12 weeks of age $(n=4$ at each age), $1 \mathrm{~g}$ masseter muscle was used for RT-PCR. Samples were scraped off and stored at $-80^{\circ} \mathrm{C}$ until used. The masseter muscle was thawed, and cut into small pieces and total RNA was extracted with a Quick Prep Total RNA Extraction Kit (Amersham Biosciences, U.K.) according to manufacturer's protocol. Contaminating DNA was removed with RNase-free DNase (DNA-free, Ambion, Austin, Texas U.S.A), and total RNA was quantified by means of spectrophotometry. The samples were stored at $-80^{\circ} \mathrm{C}$ until further use.

\section{Preparation of probes with gene primers}

The gene primers used are shown in Table 1.

mRNA Analysis by RT-PCR

The DNA oligonucleotide primers were selected from the published nucleotide sequences for each

Table 1. Oligonucleotide primers used for RT-PCR

\begin{tabular}{|c|c|c|c|c|}
\hline & & Sequence & Product(bp) & GeneBank \\
\hline ND1 & $\begin{array}{l}\text { forward } \\
\text { reverse }\end{array}$ & $\begin{array}{l}\text { 5'-AACACTCCTAATCCCAATCT-3' } \\
\text { 5'-TTGTTTCTGCGAGGGTTGAA-3' }\end{array}$ & 550 & X07479 \\
\hline $51 \mathrm{kDa}$ & $\begin{array}{l}\text { forward } \\
\text { reverse }\end{array}$ & $\begin{array}{l}\text { 5'-AACCTCATTTGGCTCGCTGA-3' } \\
\text { 5'-GCATTCTTGCCAATCAGACC-3' }^{\prime}\end{array}$ & 466 & *NM_007103, M58607 \\
\hline $75 \mathrm{kDa}$ & $\begin{array}{l}\text { forward } \\
\text { reverse }\end{array}$ & $\begin{array}{l}\text { 5'-GCAGAGCCTCACAGACAATG-3' } \\
\text { 5'-TGGGTAAGAGCACTGACTGC-3' }\end{array}$ & 451 & XM_217417 \\
\hline MyHC-I & $\begin{array}{l}\text { forward } \\
\text { reverse }\end{array}$ & $\begin{array}{l}\text { 5'-ACGGAGGAAGACAGGAAGAACCTAC-3' } \\
\text { 5'-GGGCTTCACAGGCATCCTTAG-3' }^{\prime}\end{array}$ & 288 & X15939 \\
\hline MyHC-IIa & $\begin{array}{l}\text { forward } \\
\text { reverse }\end{array}$ & $\begin{array}{l}\text { 5'-TATCCTCAGGCTTCAAGATT-3' } \\
5^{\prime} \text {-TAAATAGAATCACATGGGGACA-3' }\end{array}$ & 290 & L13606 \\
\hline MyHC-IIb & $\begin{array}{l}\text { forward } \\
\text { reverse }\end{array}$ & $\begin{array}{l}\text { 5'-CTGAGGAACAATCCAACGTC-3' } \\
\text { 5'-TTGTGTGATTTCTTCTGTCACCT-3' }\end{array}$ & 190 & L24897 \\
\hline MyHC-IId/X & $\begin{array}{l}\text { forward } \\
\text { reverse }\end{array}$ & $\begin{array}{l}\text { 5'-CTGGAGGTTCACACCAAA-3' } \\
\text { 5'-TCCCAAAGTCGTAAGTACAAAATGG-3' }\end{array}$ & 120 & AF157005 \\
\hline GAPDH & $\begin{array}{l}\text { forward } \\
\text { reverse }\end{array}$ & $\begin{array}{l}\text { 5'-ACCACAGTCCATGCCATCAC-3' } \\
5^{\prime} \text {-CCACCACCCTGTTGCTGTA-3' }\end{array}$ & 452 & M17701 \\
\hline
\end{tabular}

\footnotetext{
*: Rat 51kDa was homology of sequence based on information of homo sapiens and bovine.
} 
a

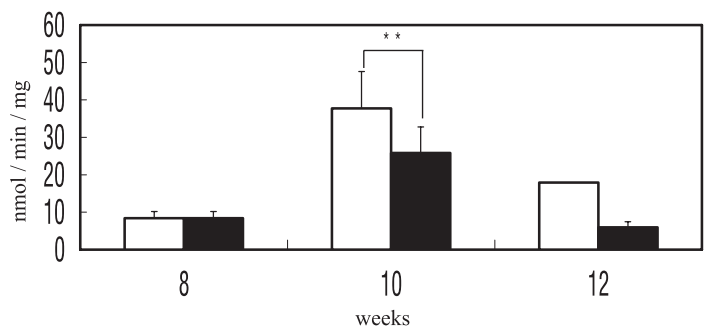

b

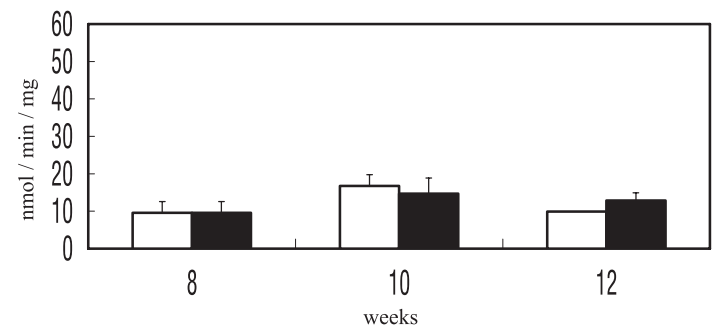

Fig. 3. Changes in enzyme activity in mitochondria isolated from rat masseter muscle for 4 weeks of feeding: a, NADH- $\mathrm{O}_{2}$ oxydoreductase in superficial portion; b, NADH-O 2 oxydoreductase in the deep portion (Columns: White, Group S; Black, Group $\mathrm{S}-\mathrm{H}) .{ }^{* *} \mathrm{p}<0.05$ for comparison between the Group S-H and the Group S.

gene (Table 1). A $1 \mu \mathrm{g}$ sample of total RNA was reverse transcribed in a $25 \mu \mathrm{l}$ volume of the reaction mixture at $50^{\circ} \mathrm{C}$ for $1 \mathrm{hr}$. The reaction mixture consisted of $0.4 \mu \mathrm{M}$ oligo (dT) 15 primer, $1 \mathrm{mM}$ dNTP, 20 units of RNase inhibitor (TaKaRa), 2.5 units of AMV reverse transcriptase (TaKaRa), $25 \mathrm{mM}$ Tris- $\mathrm{HCl}$ (pH8.3), $50 \mathrm{mM} \mathrm{KCl}, 2 \mathrm{mM}$ DTT and $5 \mathrm{mM} \mathrm{MgCl}$. As a negative control, a total RNA sample to which no reverse transcriptase had been added was tested for DNA contaminants. For PCR, $1 \mu \mathrm{l}$ of the cDNA mixture was added to $25 \mu \mathrm{l}$ of master mix consisting of $200 \mu \mathrm{M}$ dNTP, $0.4 \mu \mathrm{M}$ each of forward primer and reverse primer and 0.625 units of Ampli Taq Gold (Applied Biosystems, Foster, CA). The reactions were performed at different annealing temperatures and cycle repetitions: $60^{\circ} \mathrm{C}, 40$ cycles for GAPDH in Complex I; $60^{\circ} \mathrm{C}, 40$ cycles for ND1; $55^{\circ} \mathrm{C}, 40$ cycles for $51 \mathrm{kDa} ; 60^{\circ} \mathrm{C}, 40$ cycles for $75 \mathrm{kDa} ; 60^{\circ} \mathrm{C}, 28 \mathrm{cy}-$ cles for GAPDH in $\mathrm{MyHC} ; 60^{\circ} \mathrm{C}, 35$ cycles for MyHC-I; $60^{\circ} \mathrm{C}, 35$ cycles for MyHC-IIa; $55^{\circ} \mathrm{C}, 35$ cycles for MyHC-IIb; $55^{\circ} \mathrm{C}, 35$ cycles for MyHC$\mathrm{IId} / \mathrm{x}$. An aliquot of each PCR product was separated on a $1.5 \%$ agarose gel and stained with ethidium bromide. The stained gels were photographed under an ultraviolet illuminator with Polaroid film. The intensity of the bands was quantified with Image Gause software (Fuji Film, Tokyo, Japan).

\section{Statistical analysis}

Data are reported as means \pm SD. The statistical analysis was performed with commercially available software (Microsoft ${ }^{\circledR}$ office Excel 2003). Differences between rat masseter muscle in Group $\mathrm{S}$ and Group S-H were analyzed for significance by the t-test. Correlations between the values of groups were evaluated by calculating the best fit based on a least-squares regression analysis. The regression lines were then represented and the coefficient of correlation (R) was indicated. Significance in the statistical analyses was accepted at $\mathrm{p}<0.05$ and $\mathrm{p}<0.01$.

\section{Results}

Mitochondrial enzyme activity from 8 weeks to 12 weeks of age in the soft diet group (Group S) and the soft-hard diet group (Group S-H)

The activity patterns of $\mathrm{NADH}-\mathrm{O}_{2}$ oxidoreductase in the superficial and deep portions of the masseter muscle in both groups are shown in Fig. 3. The pattern of activity and peak activity occurred at 10 weeks of age in the superficial portion of the muscle. At 10 weeks of age, its activity in the Group $\mathrm{S}$ was higher than in the Group S-H (Fig. 3a). In contrast, $\mathrm{NADH}-\mathrm{O}_{2}$ oxidoreductase activity in the deep portion of the muscle was lower than in the superficial portion of the masseter muscle, and the pattern of activity from 8 weeks to 12 weeks of age was similar in both groups (Fig. 3b).

Muscle fiber type according to enzyme activity from 8 weeks to 12 weeks of age in the soft diet group (Group S) and the soft-hard diet group (Group S-H)

The 3 types of muscle fibers (Type I, strong reaction; Type IIA, intermediate reaction; Type IIB, weak reaction) were classified in the superficial and deep portions of the masseter muscle by the staining for NADH enzyme activity (Fig. 4). The proportion of Type I fibers in the superficial portion and deep portion was low in both groups (Figs. 5a-d), but the proportion of Type I fibers in the superficial portion in the Group S-H increased from 8 weeks to 12 weeks of age (Fig. 5b). The proportion of Type IIB fibers in both portions gradually increased from 10 weeks onward in the Group S-H (Figs. 5b, d). 


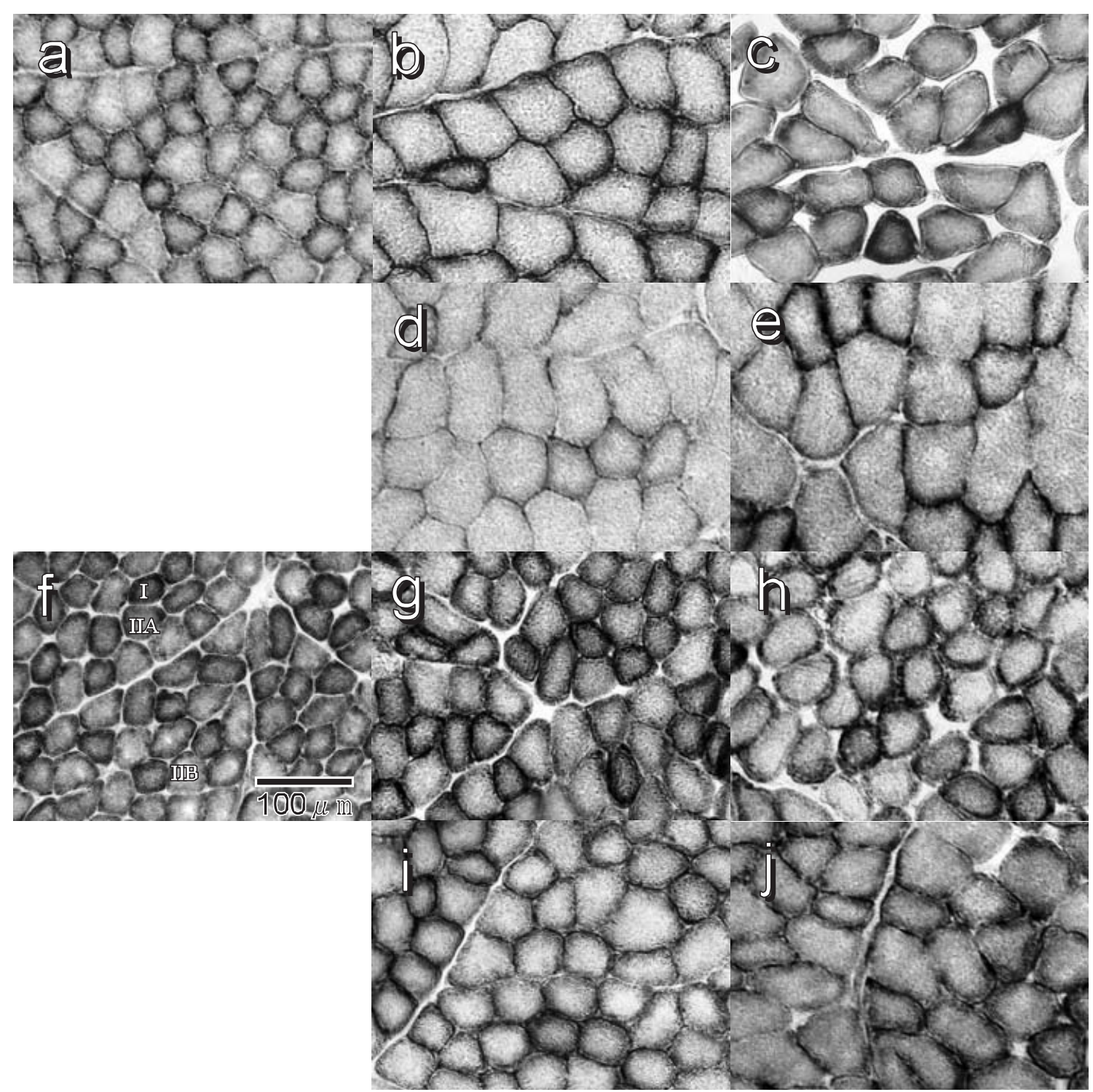

Fig. 4. Histochemical reactions in rat masseter muscle. Muscle fibers were reacted with NADH. Muscle fibers were classified into 3 types: Type I, strong reaction; Type IIA, intermediate reaction; Type IIB, light reaction. Superficial portion (a, Group S at 8 weeks; b, Group S at 10 weeks; c, Group S at 12 weeks; d, Group S-H at 10 weeks; e, Group S-H at 12 weeks); Deep portion (f, Group S at 8 weeks; g, Group S at 10 weeks; h, Group S at 12 weeks; i, Group S-H at 10 weeks; j, Group S-H at 12 weeks).

Diameter of the 3 types of muscle fibers (Type I, strong reaction; Type IIA, intermediate reaction; Type IIB, weak reaction)

The diameter of the Type IIB fibers in the superficial portion of both groups was highest of all at 10 weeks and 12 weeks of age (Fig. 6c). The diameter of all three fiber types in the superficial portion was higher in the Group S than in the Group S-H (Figs. $6 \mathrm{a}-\mathrm{c})$, whereas the diameter in the deep portion was slightly higher in the Group S-H than in the Group $\mathrm{S}$ at 10 weeks and 12 weeks of age (Figs. 6d-f).
Expression of $m R N A s$ for ND1, $51 \mathrm{kDa}$ and $75 \mathrm{kDa}$ from 8 weeks to 12 weeks of age in the soft diet group (Group $S$ ) and the soft-hard diet group (Group S-H)

The expression pattern of mRNAs for Complex I components (ND1, $51 \mathrm{kDa}$ and $75 \mathrm{kDa}$ ) was shown in Fig. 7(I). At 12 weeks of age, the levels of the ND1 and $75 \mathrm{kDa}$ mRNAs in the superficial portion of the masseter muscle were higher in the Group S-H than in the Group S (Figs. 7(II) a, c). The level of $51 \mathrm{kDa}$ mRNA in the superficial portion in the 

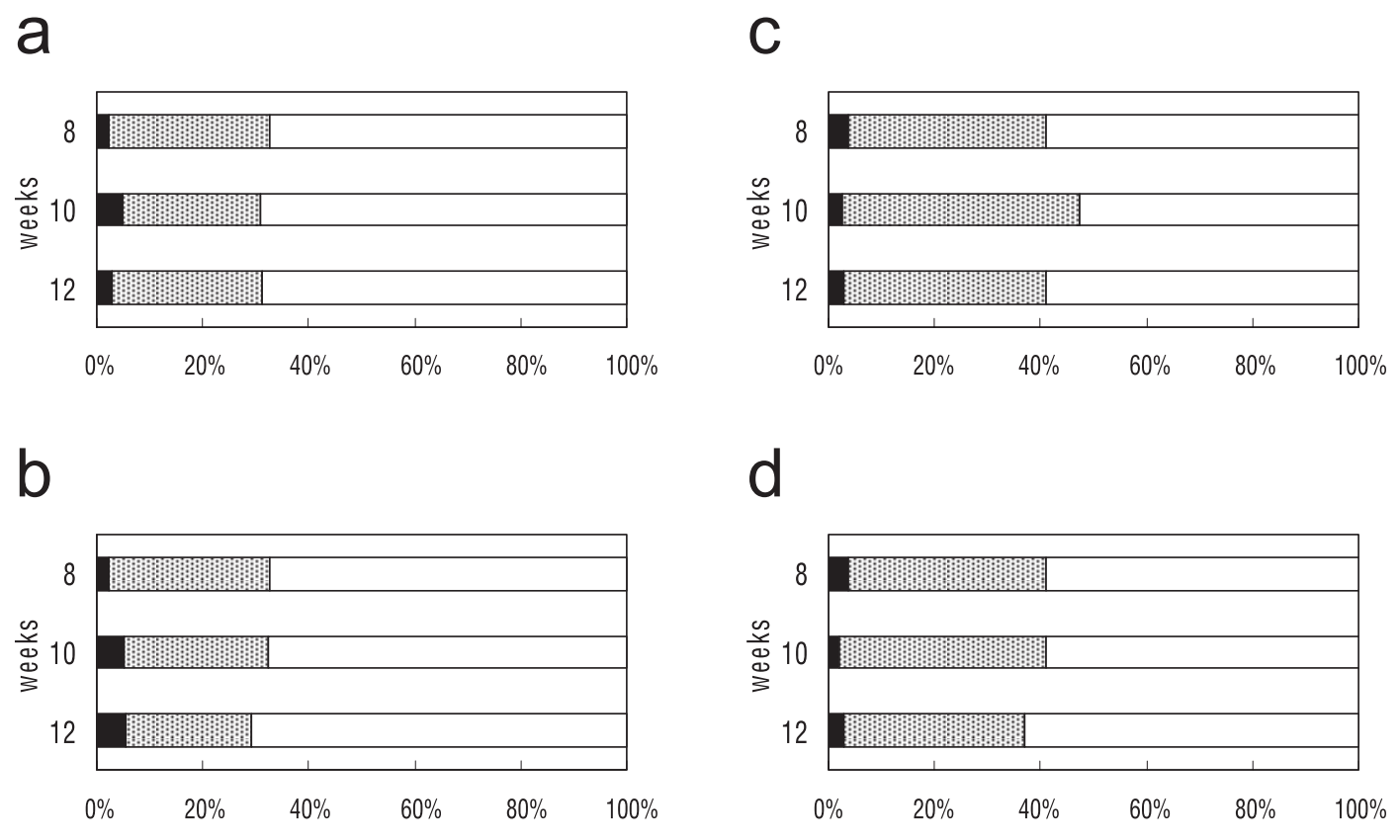

Fig. 5. Proportions of muscle fiber types (Type I, IIA and IIB): Superficial portion (a, Group S; b, Group S-H); Deep portion (c, Group S; d, Group S-H) (Columns: Black, Type I; Gray, Type IIA; White, Type IIB).

Group S was markedly highest at 10 weeks of age (Fig. 7(II) b). At 12 weeks of age, the levels of ND1, 51kDa and $75 \mathrm{kDa}$ mRNAs in the deep portion in the Group S-H were higher than in the Group S (Figs. 7(II) d-f).

Expression of mRNAs for MyHC-I, MyHC-IIa, MyHC-IIb and MyHC-IId/x from 8 weeks to 12 weeks of age in the soft diet group (Group $S$ ) and the soft-hard diet group (Group $\mathrm{S}$-H)

The MyHCs (MyHC-I, MyHC-IIa, MyHC-IIb and MyHC-IId/x) mRNA levels were higher in the superficial portion than in the deep portions in both groups (Figs. 8 (II) a-h). In particular, the levels of MyHC-IIa and MyHC-IId/x mRNAs in the superficial portion of the masseter muscle in the Group $\mathrm{S}-\mathrm{H}$ were high at 12 weeks of age (Fig. 8 (II) b, d). The level of MyHC-I mRNA in the superficial portion of Group S was high at 10 weeks (Fig. 8 (II) a). The MyHCs mRNA levels in the deep portion were almost no difference between Group S and Group S-H.

\section{Discussion}

The stress caused by feeding a soft diet gradually affects enzyme activity in the masseter muscle during mastication and leads to the observed changes in metabolic activity. In general, a soft diet feeding effect to the mechanism of mastication by changing occlusion $^{7,9,10,16,17)}$. We have already shown decreased levels of succinate- $\mathrm{O}_{2}$ oxidoreductase and $\mathrm{NADH}-\mathrm{O}_{2}$ oxidoreductase activity in the masseter muscle during the early stages of feeding a soft diet, with a clear nadir at 60 days $^{18}$. The myosin ATPase activity of guinea pigs decreases significantly after bite opening at 1 week $^{22)}$. The timing of the decreases in enzyme activity suggests differences in resistance to feeding stress according to soft diets. However, there have been no studies on variations in enzyme activity with respect to the anatomical structure of the superficial and deep portions of the masseter muscle in animals fed a soft diet. Our results showed increased enzyme activity in the superficial portion of the masseter muscle in both the group S-H and group S at 10 weeks of age (70 days), and it had decreased at 12 weeks. Thus, different enzyme activity was found in the superficial and deep portions of the masseter muscle on our experimental diets, indicating that the superficial portion of the rat masseter muscle is more easily affected than the deep portion, and then recovers in the superficial portion as the mastication system adapts to the mechanical stress.

The percentages of Type IIA fibers and Type IIB fibers in the superficial portion of the masseter muscle of rats decreased for a period of 2 weeks under stress with protrusion of mandible ${ }^{7}$. After 
a

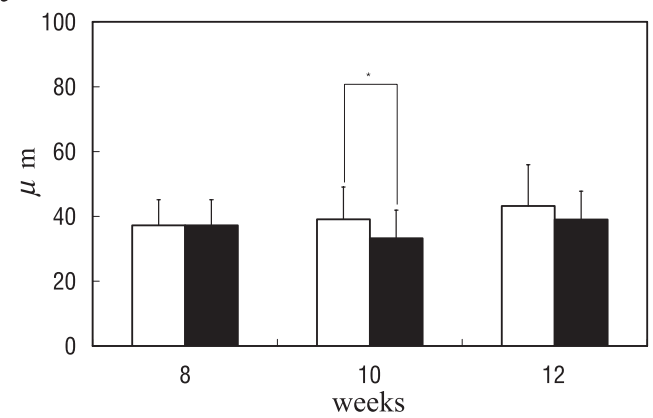

b

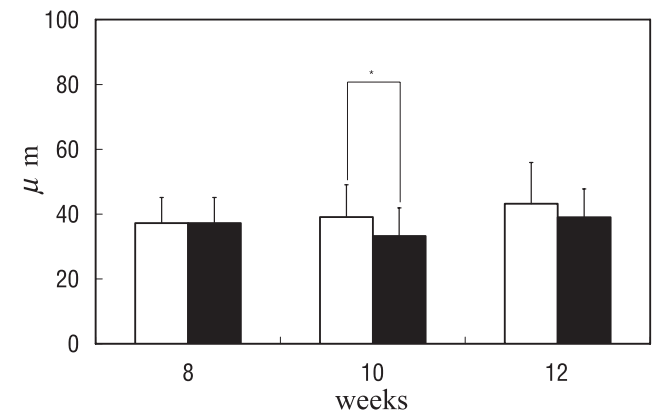

C

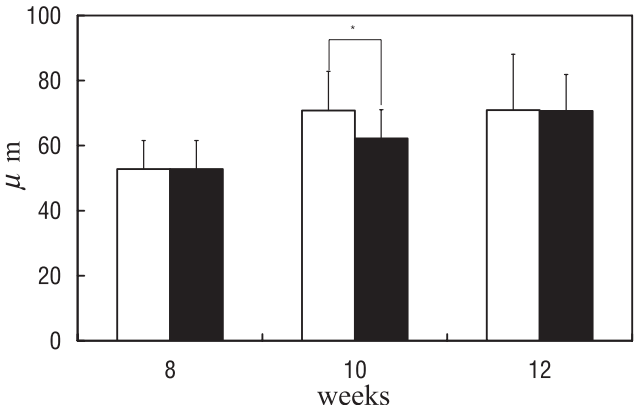

d

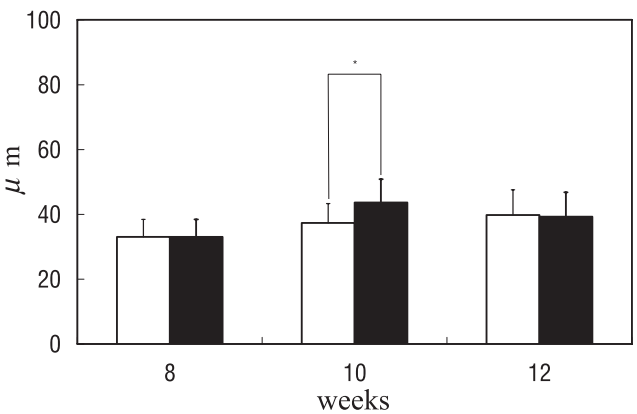

e

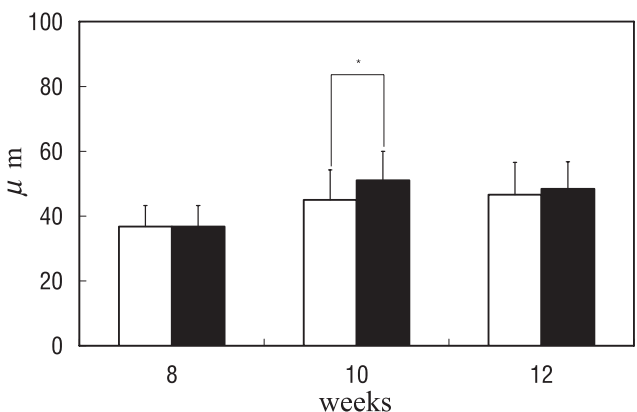

f

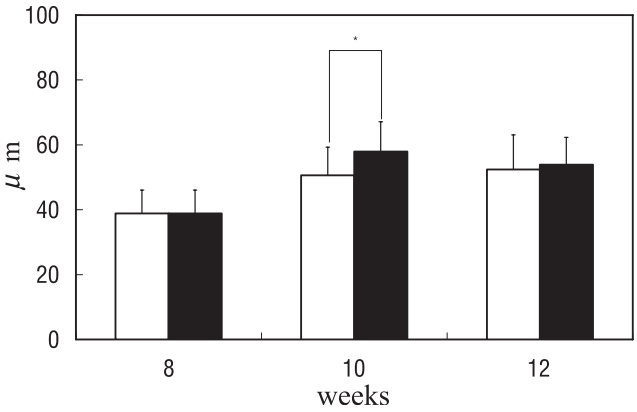

Fig. 6. Diameter of muscle fibers in rat masseter muscle: Superficial portion (a, Type I; b, Type IIA; c, Type IIB); Deep portion (d, Type I; e, Type IIA; f, Type IIB) (Columns: White, Group S; Black, Group S-H). *p $<0.01$ for comparison between the Group S-H and Group S.

bilateral extraction of lateral teeth, Type IIB fiber with succinate dehydrogenase staining was low in the deep portion of the masseter muscle of rats fed a soft diet $^{9)}$. In contrast, Kiliaridis et al. ${ }^{6)}$ reported that different compositions of muscle fiber types were found in animals fed between a soft diet and a hard diet for a short period. There were a smaller percentage of Type IIA fibers and a larger percentage of Type IIB fibers in the anterior deep masseter muscle in the group fed a soft diet for 28 days than in with the group fed a normal diet. Our results showed that the proportion of Type IIB fibers in the superficial portion and deep portion of the Group S-H increased from 8 weeks to 12 weeks of age even though the proportions of both re- mained unchanged in the Group S. The differences proportion of Type IIB fibers of in the Group S in our study and the study by Kiliaridis et al. ${ }^{6}$ may be due to different portions of masseter muscle that were examined. Muscle fiber size changed somewhat in response to dietary stress for 4 weeks, and muscle fiber size did not appear to reflect the functional demand in enzyme activity caused by changes in food consistency. Kitagawa et al. ${ }^{23)}$ also suggested that the proportion of Type I fibers in the deep portion of the rabbit masseter muscle decreased after long-term feeding a soft diet for 6 months, even though there was no difference in fiber size between the control group and soft-diet group. Our results suggest that a change from soft 
(I)

\section{superficial portion}

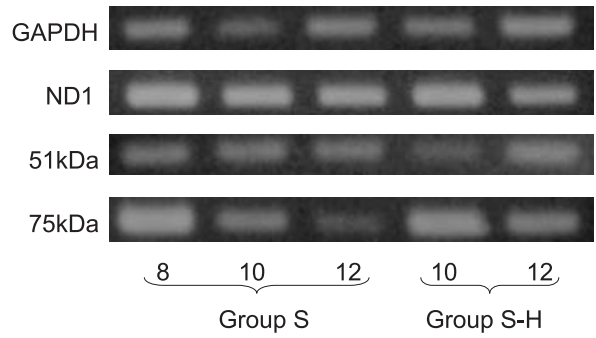

(II)

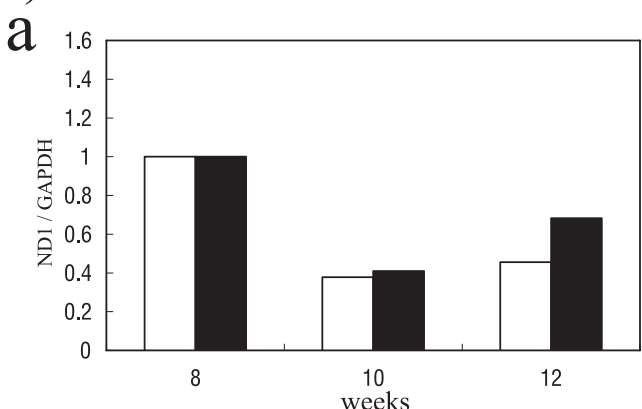

b
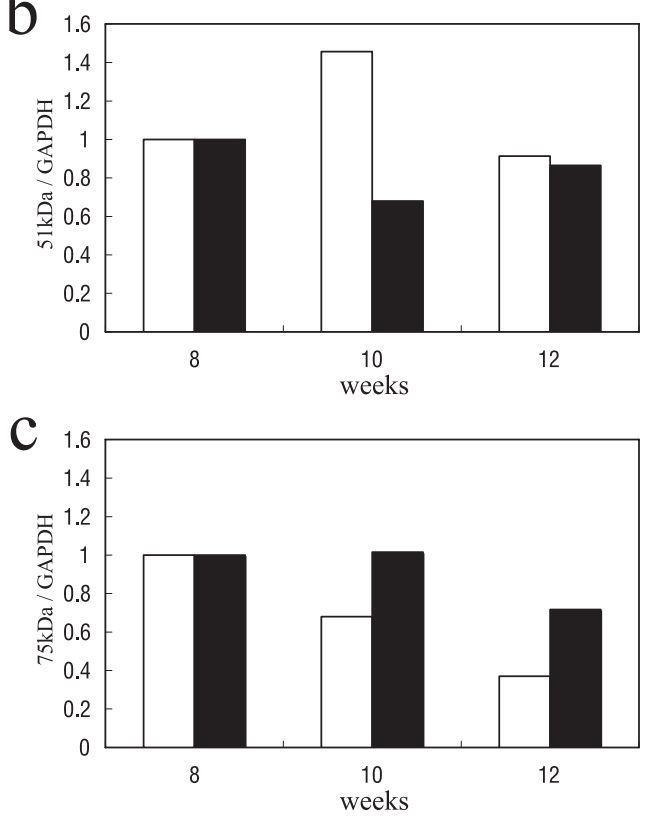

deep portion
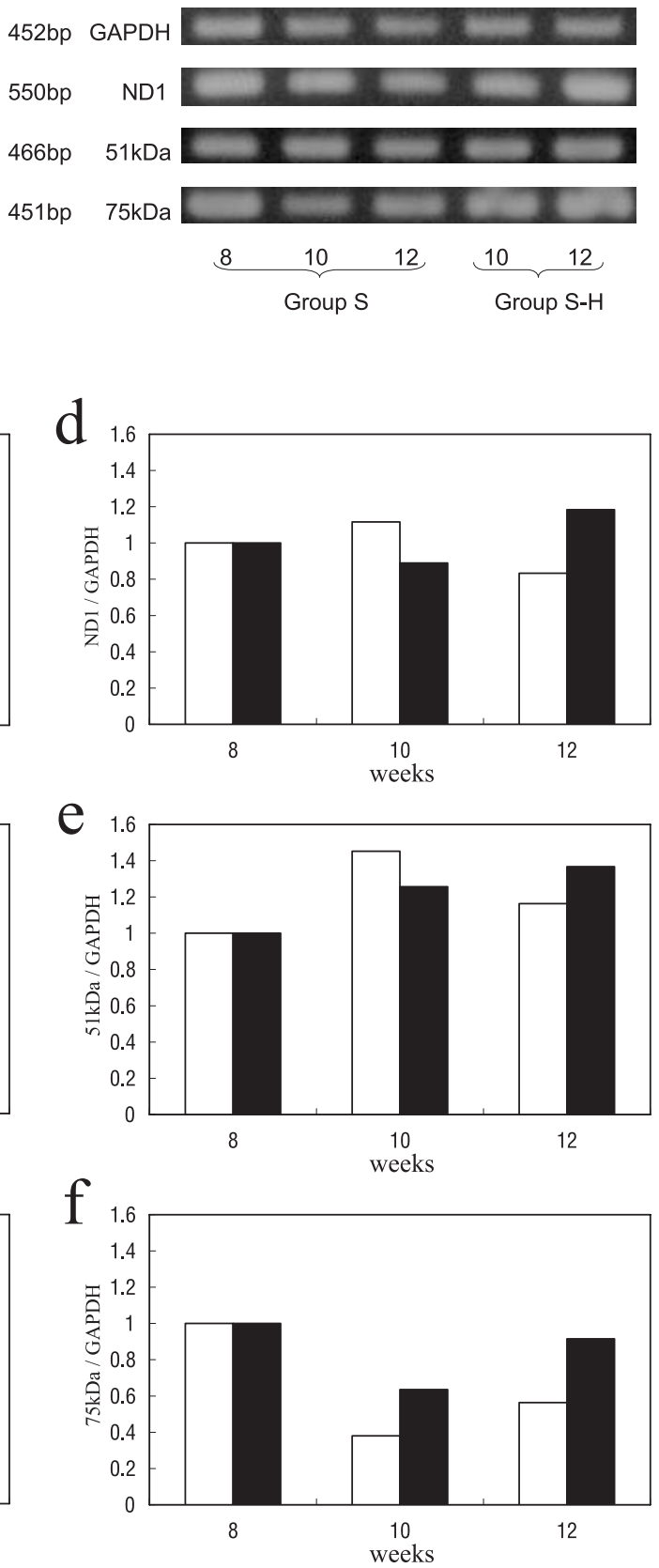

Fig. 7. RT-PCR of ND1, 51kDa, $75 \mathrm{kDa}$ and GAPDH mRNA in rat masseter muscle (Superficial portion, Deep portion).

(I) Expressions of mRNA (ND1, 51kDa, 75kDa and GAPDH) in rat masseter muscle.

(II) Relative intensity was obtained from the image analysis of RT-PCR: Superficial portion (a, ND1; b, 51kDa; c, 75kDa); Deep portion (d, ND1; e, 51kDa; f, 75kDa) (Columns: White, Group S; Black, Group S-H).

diet to hard diet feeding induces changes in enzyme activity, fiber phenotype and muscle fiber size, and indicate that 4 weeks of stress leads to different adaptations of the masseter muscle compared to those seen during long-term feeding of growing rats with a soft diet, extraction of teeth treatments, and opening bite feeding conditions.

Our focus was the level of NADH-ubiquinone 
(I)

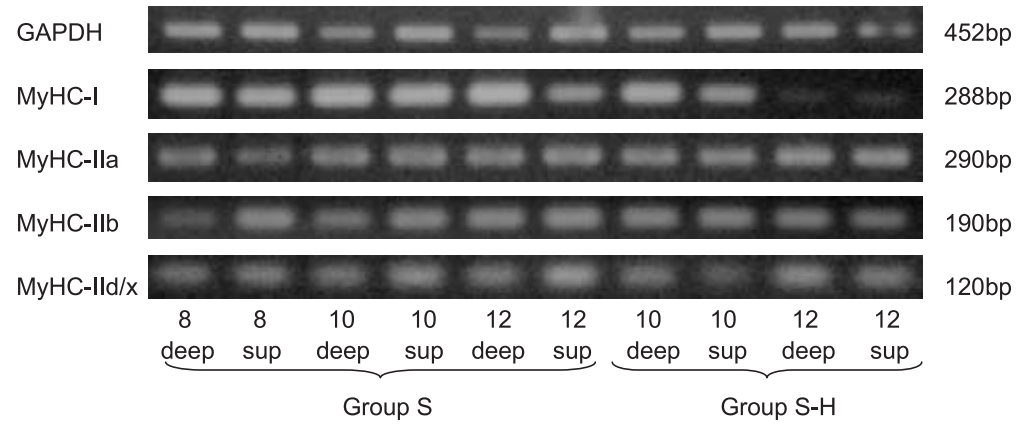

(II)
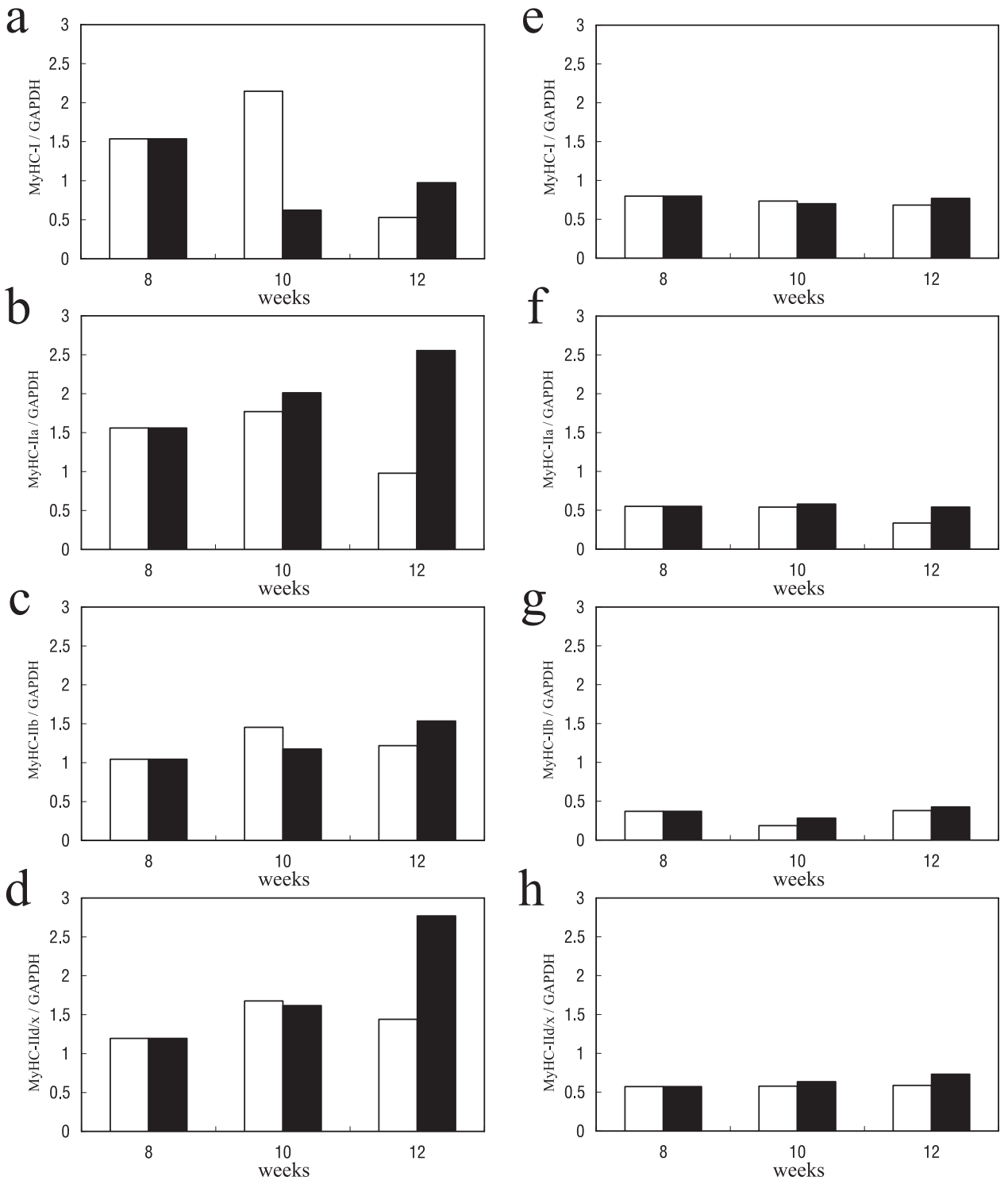

Fig. 8. RT-PCR of MyHC-I, MyHC-IIa, MyHC-IIb, MyHC-d/x and GAPDH mRNA in rat masseter muscle (Superficial portion, Deep portion).

(I) Expressions of mRNA (MyHC-I, MyHC-IIa, MyHC-IIb, MyHC-d/x and GAPDH) in rat masseter muscle.

(II) Relative intensity was obtained by image analysis of RT-PCR: Superficial portion (a, MyHC-I; b, MyHC-IIa; c, MyHC-IIb; d, MyHC-IId/x); Deep portion (e, MyHC-I; f, MyHC-IIa; g, MyHC-IIb; h, MyHC-IId/x) (Columns: White, Group S; Black, Group S-H). 
oxidoreductase (Complex I), which is encoded by the nuclear genome (34 encoded subunits) and the mitochondrial genome ( 7 encoded subunits). The ND1 subunit is the terminal acceptor for quinone reduction within the membrane portion of Complex $\mathrm{I}^{24)}$. The $51 \mathrm{kDa}$ and $75 \mathrm{kDa}$ subunits are involved in electron transfer to $\mathrm{NADH}^{25}$. ND1 is encoded in the mitochondria, whereas $51 \mathrm{kDa}$ and $75 \mathrm{kDa}$ are encoded in the nucleus and may be rate-limiting for enzyme formation. The mRNA levels of these enzymes differed both between the superficial and deep portions of the masseter muscle and according to experimental conditions such as feeding a soft diet or a hard diet. Our results showed a difference in expression of mRNA between Group $\mathrm{S}$ and Group S-H at 10 weeks and 12 weeks of age. The $51 \mathrm{kDa}$ mRNA expression in the masseter muscle was high in the Group S, and it correlated with the high NADH oxidoreductase enzyme activity from 8 weeks to 10 weeks. During postnatal development of the rat masseter muscle, expression of the ND1 and 51kDa mRNAs differs depending on feeding behavior from suckling to mastication ${ }^{13)}$. The $51 \mathrm{kDa}$ subunit is rate-limiting for formation of NADH-ferricyanide reductase during development of rat tongue muscle ${ }^{14}$. In the present study, $51 \mathrm{kDa}$ mRNA levels in the superficial portion were also related to enzyme activity during stress caused by a soft diet, at least during the first 2 weeks. In contrast, expression of ND1 and $75 \mathrm{kDa}$ mRNAs was high in changing feeding behavior, such as when they changed from a soft diet to a hard diet for 4 weeks. Therefore after 4 weeks of stress on the hard diet, the high levels of mRNA expression of these subunits suggest that feeding a hard diet may act as a positive induces of the formation of NADH-ferricyanide reductase. Expression of the $75 \mathrm{kDa}$ subunit may be controlled by the expression level of the $51 \mathrm{kDa}$ subunit, which are related to the rate-limiting for formation of NADH-ferricyanide reductase during soft diet feeding stress, because the nuclear-encoded $75 \mathrm{kDa}$ subunit is downstream of the electron transfer to NADH. Therefore, the change a soft diet to hard diet may not cause any changes in enzyme activity.

Sugiura et al. ${ }^{26)}$ reported changes in the expression of MyHC isoforms during 5 weeks of overloading stress on skeletal muscle. Ohnuki et al. ${ }^{16)}$ reported an increase in MyHC-IIa mRNA and a decrease in MyHC-IIb mRNA in the masseter muscle over a 1-week period during bite opening in rats. However, these different MyHC mRNA levels may reflect muscle-specific profiles and different stress between skeletal muscle and masticatory muscle. In the present study, a decrease in MyHC-I mRNA was found in the superficial portion of the masseter muscle of the Group S-H at 10 weeks of age. Expression of the MyHC-I, MyHC-IIa, MyHC$\mathrm{IIb}$ and MyHC-IId/x mRNAs in the superficial portion in the Group S-H was highest in 12 weeks of age, reflecting a positive reaction to the stress induced by the change in diet. The change from soft diet to hard diet also caused changes in the mRNAs for the Complex I subunits in 12-week-old rats, but these changes were not reflected in the muscle phenotype after 4 weeks on the hard diet, because there were marked no changes after 2 weeks or 4 weeks of feeding. This suggests that feeding stress caused by a change from soft diet to hard diet affects oxidative metabolism and the expression of mRNAs encoding Complex I subunits and MyHC isoforms, but has no clear effect on muscle phenotype. These results should be compared with the observation of MyHC changes after strong stretch overloading caused by bite opening ${ }^{16)}$ or with the different composition of masseter muscle fiber types in soft-diet-fed and hard-diet-fed animals, as reported in rats by Kiliaridis et al. ${ }^{6)}$ and in rabbits by Kitagawa et al. ${ }^{23)}$

\section{References}

1) Bredman JJ, Weijs WA and Moorman AF. Myosin heavy chain expression in rabbit masseter muscle during postnatal development. J Anat 1992; 180 (Pt 2):263-274.

2) Fujimoto S, Watanabe J, Ogawa R and Kanamura S. Agerelated changes in fibre number, fibre size, fibre type composition and adenosine triphosphatase activity in rat soleus muscle. Anat Anz 1994; 176:429-435.

3) Sato I, Konishi K, Kuramochi $T$ and Sato T. Developmental changes in enzyme activities and in structural features of rat masticatory muscle mitochondria. J Dent Res 1998; 77:1926-1930.

4) Agbulut $\mathrm{O}$, Noirez P, Beaumont $\mathrm{F}$ and Butler-Browne G. Myosin heavy chain isoforms in postnatal muscle development of mice. Biol Cell 2003; 95:399-406.

5) Guelinckx P, Dechow PC, Vanrusselt R and Carlson DS. Adaptations in the temporalis muscles of rabbits after masseter muscle removal. J Dent Res 1986; 65:1294-1299.

6) Kiliaridis S, Engstrom C and Thilander B. Histochemical analysis of masticatory muscle in the growing rat after prolonged alteration in the consistency of the diet. Arch Oral Biol 1988; 33:187-193.

7) Easton JW and Carlson DS. Adaptation of the lateral pterygoid and superficial masseter muscles to mandibular protrusion in the rat. Am J Orthod Dentofacial Orthop 1990; 97:149-158.

8) Miyata H, Sugiura $T$, Kawai $Y$ and Shigenaga $Y$. Effect of soft diet and aging on rat masseter muscle and its motoneuron. Anat Rec 1993; 237:415-420.

9) Miehe B, Fanghanel J, Kubein-Meesenburg D, Nagerl H and Schwestka-Polly R. Masticatory musculature under altered occlusal relationships - a model study with experimental animals. Anat Anz 1999; 181:37-40.

10) Nishide $\mathrm{N}$, Baba $\mathrm{S}$, Hori $\mathrm{N}$ and Nishikawa H. Histological study of rat masseter muscle following experimental occlusal alteration. J Oral Rehabil 2001; 28:294-298. 
11) Bresin A, Bagge $U$ and Kiliaridis S. Adaptation of normal and hypofunctional masseter muscle after bite-raising in growing rats. Eur J Oral Sci 2000; 108:493-503.

12) Weiss H, Friedrich T, Hofhaus G and Preis D. The respiratory-chain NADH dehydrogenase (complex I) of mitochondria. Eur J Biochem 1991; 197:563-576.

13) Sato I, Sunohara $M$ and Kunimoto $S$. Expressions of mRNA ND1 and 51k in Mitochondria of Postnatal Rat Masseter Muscle. Dent Jpn 2003; 39:3-7.

14) Fujita $\mathrm{T}$ and Sato I. NADH-O $\mathrm{O}_{2}$ oxidoreductase activity and mRNA expression of complex I $(51 \mathrm{kDa}, \mathrm{ND} 1)$ in postnatal intrinsic muscle of rat tongue. J Anat 2003; 202:205-212.

15) Widmer CG, Morris-Wiman JA and Nekula C. Spatial distribution of myosin heavy-chain isoforms in mouse masseter. J Dent Res 2002; 81:33-38.

16) Ohnuki Y, Saeki Y, Yamane A and Yanagisawa K. Quantitative changes in the mRNA for contractile proteins and metabolic enzymes in masseter muscle of bite-opened rats. Arch Oral Biol 2000; 45:1025-1032.

17) Cvetko E and Erzen I. Effect of chronic uncontrollable stress on myosin heavy chain expression in rat masseter muscle. Pflugers Arch 2001; 442 (Suppl):R147-149.

18) Sato I and Konishi K. Effects of soft diet on rat masseter muscle mitochondrial development. Okajimas Folia Anat Jpn 2004; 81:67-74.

19) Novikoff AB, Shin WY and Drucker J. Mitochondrial localization of oxidative enzymes: staining results with two tetrazolium salts. J Biophys Biochem Cytol 1961; 9:47-61.
20) Hogeboom GH. Fractionation of cell components of animal tissues, Methods in Enzymology; in Colowick, SP, NO Kaplan (eds). Preparation and Assay of Enzymes. New York: Academic Press 1955; 1:16-19.

21) Lowry OH, Rosebrough NJ, Farr AL and Randall RJ. Protein measurement with the Folin phenol reagent. J Biol Chem 1951; 193:265-275.

22) Kawasaki K, Saeki Y and Ohnuki Y. Effect of an increase in occlusal vertical dimension on the rate of cyclic actinmyosin interaction in guinea-pig masseter muscle. Arch Oral Biol 1997; 42:505-512.

23) Kitagawa Y, Mitera K, Ogasawara T, Nojyo Y, Miyauchi K and Sano K. Alterations in enzyme histochemical characteristics of the masseter muscle caused by long-term soft diet in growing rabbits. Oral Dis 2004; 10:271-276.

24) Ragan CI, Galante YM, Hatefi Y and Ohnishi T. Resolution of mitochondrial NADH dehydrogenase and isolation of two iron-sulfur proteins. Biochemistry 1982; 21:590-594.

25) Brandt U. Proton-translocation by membrane-bound NADH: ubiquinone-oxidoreductase (complex I) through redox-gated ligand conduction. Biochim Biophys Acta 1997; 16:79-91.

26) Sugiura $T$, Miyata $H$, Kawai $Y$, Matoba $H$ and Murakami $\mathrm{N}$. Changes in myosin heavy chain isoform expression of overloaded rat skeletal muscles. Int J Biochem 1993; 25:1609-1613. 
